\title{
The Development of Anti-windup Scheme for Time Delay Control with Switching Action using Integral Sliding Surface
}

\author{
Sung-Uk Lee, \\ sulee@mecha.kaist.ac.kr,
}

\author{
Pyung Hun Chang, \\ phchang@kaist.ac.kr,
}

\author{
Je Hyung Jung \\ gory@mail.kaist.ac.kr
}

Department of Mechanical Engineering,

Korea Advanced Institute of Science and Technology,

373-1 Kusung-dong, Taejon 305-701, Korea

\begin{abstract}
The Time Delay Control with Switching Action (TDCSA) method, which consists of Time Delay Control(TDC) and a switching action of sliding mode control(SMC), has been proposed as a promising technique in the robust control area, where the plant has unknown dynamics with parameter variations and substantial disturbances are preset. When TDCSA is applied to the plant with saturation nonlinearity, however, the so-called windup phenomena are observed to arise, causing excessive overshoot and instability. The integral element of TDCSA and the saturation element of a plant cause the windup phenomena. There are two integral effects in TDCSA. One is the integral effect occurred by time delay estimation of TDC. Other is the integral term of an integral sliding surface. In order to solve this problem, we have proposed an antiwindup scheme method for TDCSA. The stability of the overall system has been proved for a class of nonlinear system. Experiment results show that the proposed method overcomes the windup problem of the TDCSA.
\end{abstract}

Keywords Windup, Time Delay Control, Switching Action

\section{INTRODUCTION}

Recently the Time Delay Control with Switching Action(TDCSA) method[1][6][7] has been proposed as a promising technique in the robust control area, where the plant has unknown dynamics with parameter variations and substantial disturbances are preset. More specifically, TDCSA consists of TDC, which estimates the amounts of an unknown nonlinear dynamics and unexpected uncertainties and cancels it, and a switching action based on sliding mode control. The switching action, the discontinuous input of sliding mode control, keeps the tracking errors, which is occurred by the time delay estimation error, on the predefined sliding surface, thereby improving the robustness. Its effectiveness has been demonstrated through the successful application to a 21-ton robotic excavator[6], a DC motor[1] and a linear motor[7].

We have observed, however, that in the presence of the saturation, TDCSA using an integral sliding surface reveals some problems commonly found in other methods like PID control. More specifically, we have observed the following problems: as we increase the command input or the response speed, there arise large overshoots, limit cycles, or even unstable responses. For this reason, we have investigated on their causes and tried to find out effective remedies. As will be explained in Section 3, we found that this phenomenon comes from the inherent integral effect in TDCSA using an integral sliding surface due to the timedelay term and an integral term of sliding surface. Specifically, the large overshoot arises from the integral effect combined with a saturation element in the system. Incidentally, this phenomenon, widely known as windup phenomenon, has been observed in the PI or PID control systems.
This windup phenomenon is occurred in the sliding mode control(SMC) using an integral sliding surface[3][5][8], like TDCSA using an integral sliding surface. In the presence of the saturation, SMC using an integral sliding surface reveals same problem found in the TDCSA using an integral sliding surface. More specially, as the command input or the response speed is increased, there arise large overshoots and oscillation because the integral term of the sliding surface is increased excessively. For remedy, Cho, et al. use a small integral gain[3]. For SMC using the saturation function or boundary layer, Slotine proposed the stopping integration method[8], which maintains the integral term constant as long as the system is outside the boundary layer. This method has a demerit that this method must know exactly the magnitude of the boundary layer.

We observed the windup phenomenon in TDC control system[1][2]. However, there have been no researches reporting this phenomenon in TDCSA, nor any addressing this causes, not to mention any proposing this remedy. Therefore, in this study, we provide remedy against this problem, by proposing compensator specifically designed for TDCSA using an integral sliding surface. Accordingly, the contribution we attempt to make is threefold:

- to report the existence of the windup problem and will be demonstrated through simulations and experiments;

- to provide the analysis on the causes of this; and

- to provide remedy to overcome this.

\section{TIME DELAY CONTROL WITH SWITCHING ACTION}

In this section, the TDCSA will be briefly introduced and the stability of TDCSA using an integral sliding surface is analyzed.

\subsection{TDCSA}

A class of plants we are concerned with can be expressed in the following nonlinear differential equation.

$$
\dot{\mathbf{x}}(t)=\mathbf{f}(\mathbf{x}, t)+\mathbf{B}(\mathbf{x}, t) \mathbf{u}(t)+\mathbf{d}(t)
$$

where $\mathbf{x}(t) \in R^{n}$ denotes state vector of plant; $\mathbf{u}(t) \in R^{r}$ the control input vector; $\mathbf{f}(\mathbf{x}, t) \in R^{n \times 1}$ nonlinear function in companion form, which may be unknown; $\mathbf{d}(t) \in R^{n \times 1}$ unknown disturbances; $\mathbf{B}(\mathbf{x}, t) \in R^{n \times r}$ the control distribution matrix, the range of which should be known. In this plant, it is assumed that the states and their derivatives are measurable. If we select a constant matrix $\overline{\mathbf{B}}$ which is located within the known range of $\mathbf{B}(\mathbf{x}, t)$ and use $\overline{\mathbf{B}}$, Eq. (1) is rearranged into the following:

$$
\dot{\mathbf{x}}(t)=\overline{\mathbf{B}} \mathbf{u}(t)+\mathbf{H}(t)
$$

where $\mathbf{H}(t)$ consists of terms representing uncertainties and time-varying factors, which are expressed as 


$$
\mathbf{H}(t)=\mathbf{f}(\mathbf{x}, t)+\{\mathbf{B}(\mathbf{x}, t)-\overline{\mathbf{B}}\} \mathbf{u}(t)+\mathbf{d}(t)
$$

a) Time Delay Control (TDC) [4][11]

In TDCSA control system, the control objective of TDC is to make the plant in Eq. (2) follow accurately a desired error dynamics in the presence of unknown dynamics and unexpected disturbance. The desired error dynamics is defined with a reference model, an asymptotically stable linear invariant system as the following:

$\dot{\mathbf{e}}(t)=\mathbf{A}_{\mathbf{m}} \mathbf{e}(t)$

where $\mathbf{e}(t)=\mathbf{x}_{\mathbf{d}}(t)-\mathbf{x}(t) \in R^{n}$ is tracking error vector, $\mathbf{x}_{\mathrm{d}} \in R^{n}$ is desired trajectory vector. And $\mathbf{A}_{\mathrm{m}} \in R^{n \times n}$ is a constant matrix of the desired error dynamics..

The TDC law that meets the desired error dynamics(Eq. (4)) is obtained as

$$
\mathbf{u}_{\mathrm{tdc}}(t)=\overline{\mathbf{B}}^{+}\left[\dot{\mathbf{x}}_{\mathbf{d}}(t)-\hat{\mathbf{H}}(t)-\mathbf{A}_{\mathrm{m}} \mathbf{e}(t)\right]
$$

where $\overline{\mathbf{B}}^{+}$denotes a pseudo-inverse of $\overline{\mathbf{B}}$ $\left(\bar{B}^{+}=\left(\bar{B}^{T} \bar{B}\right)^{-1} \bar{B}^{T}\right) ; \hat{\mathbf{H}}(t)$ the estimate of $\mathbf{H}(t)$. If $\mathrm{L}$ is very small and $\mathbf{H}(t)$ does not vary largely during the $\mathrm{L}$ times, the estimated $\hat{\mathbf{H}}(t)$ can be obtained by using both Eq. (2) and the fact that $\mathbf{H}(t)$ is usually a continuous function. More specifically, when $\mathrm{L}$ is small enough, then

$$
\hat{\mathbf{H}}(t) \approx \mathbf{H}(t-L)=\dot{\mathbf{x}}(t-L)-\overline{\mathbf{B}} \mathbf{u}(t-L)
$$

Combining Eq. (6) with Eq. (5), the TDC law is obtained as follows:

$$
\mathbf{u}_{\mathrm{tdc}}(t)=\mathbf{u}_{\mathrm{tdc}}(t-L)+\overline{\mathbf{B}}^{+}\left[\dot{\mathbf{x}}_{\mathrm{d}}(t)-\dot{\mathbf{x}}(t-L)-\mathbf{A}_{\mathrm{m}} \mathbf{e}(t)\right]
$$

More details about the stability condition and the design of TDC can be found in [4][11]. More specifically, if the constant matrix $\overline{\mathbf{B}}$ is satisfied with $\left\|\mathbf{I}-\mathbf{B} \overline{\mathbf{B}}^{+}\right\|<\mathbf{1}$, the system is stable.

$\mathrm{L}$ should be sufficiently small for TDC to meet the desired error dynamics of Eq. (4). The value used for L, however, is set to be that of the sampling time, when TDC is implemented in a real-time controller. The variation of system nonlinearities and disturbances, occurred during the time delay(L), cause time delay estimation(TDE) error as follows:

$$
\mathbf{H}(t)-\hat{\mathbf{H}}(t)=\mathbf{H}(t)-\mathbf{H}(t-L)=\Delta \mathbf{H}(t)
$$

\section{b) Switching action [8]}

Switching action is discontinuous input used at sliding mode control and keeps the tracking error on predefined sliding surface. The switching action used at TDCSA keeps the tracking error, which is occurred by the TDE error, on the sliding surface so that the effect of the TDE error is reduced and also the controller becomes robust.

Why selecting the integral sliding surface for switching action is to match the desired error dynamics of TDC with the derivative of the sliding surface. In order to match the desired error dynamics(Eq. (4)) with the sliding surface $(\mathbf{s}(t)=0)$, we use the integral sliding surface as follows:

$$
\mathbf{S}(t)=\overline{\mathbf{B}}^{+} \int_{0}^{t}\left[\dot{\mathbf{e}}(\tau)-\mathbf{A}_{\mathbf{m}} \mathbf{e}(\tau)\right] \mathbf{d} \tau
$$

where the sliding surface has the initial value of zero ( $\mathbf{s}(t=0)=0)$ and its derivative(Eq. (10)) is equal to the desired error dynamics(Eq. (4)).
$\dot{\mathbf{S}}(t)=\overline{\mathbf{B}}^{+}\left[\dot{\mathbf{e}}(t)-\mathbf{A}_{\mathbf{m}} \mathbf{e}(t)\right]$

c) TDCSA

Finally, the TDCSA is proposed by adding the switching action to TDC, as follows:

$$
\begin{aligned}
\mathbf{u}(t)= & \mathbf{u}(t-L)+\overline{\mathbf{B}}^{+}\left[\dot{\mathbf{x}}_{\mathbf{d}}(t)-\dot{\mathbf{x}}(t-L)-\mathbf{A}_{\mathbf{m}} \mathbf{e}(t)\right] \\
& +\mathbf{K s g n}(\mathbf{s}(t))
\end{aligned}
$$

where $\mathbf{K} \in R^{r \times r}$ denotes a switching gain matrix, which is obtained from the following stability condition; $\mathbf{s} \in R^{r}$ the sliding surface vector

\subsection{Stability Analysis of TDCSA}

For the stability analysis of the overall system, the second method of Lyapunov is used. If the Lyapunov function is selected as $\mathbf{V}=\mathbf{s}^{\mathrm{T}} \mathbf{S} / 2$, its time derivative is as follows,

$$
\begin{aligned}
\dot{\mathbf{V}} & =\mathbf{s}^{\mathrm{T}} \dot{\mathbf{s}}=\mathbf{s}^{\mathrm{T}} \overline{\mathbf{B}}^{+}\left[\dot{\mathbf{e}}(t)-\mathbf{A}_{\mathbf{m}} \mathbf{e}(t)\right] \\
& =\mathbf{s}^{\mathrm{T}} \overline{\mathbf{B}}^{+}\left[\dot{\mathbf{x}}_{\mathbf{d}}(t)-\overline{\mathbf{B}} \mathbf{u}(t)-\mathbf{H}(t)-\mathbf{A}_{\mathbf{m}} \mathbf{e}(t)\right] \\
& =\mathbf{s}^{\mathrm{T}} \overline{\mathbf{B}}^{+}\left[\dot{\mathbf{x}}_{\mathbf{d}}(t)-\overline{\mathbf{B}}\left\{\overline{\mathbf{B}}^{+}\left(\dot{\mathbf{x}}_{\mathbf{d}}(t)-\hat{\mathbf{H}}(t)-\mathbf{A}_{\mathbf{m}} \mathbf{e}(t)\right)\right.\right. \\
& \left.+\mathbf{K} \operatorname{sgn}(\mathbf{s})\}-\mathbf{H}(t)-\mathbf{A}_{\mathrm{m}} \mathbf{e}(t)\right] \\
& =\mathbf{s}^{\mathrm{T}} \overline{\mathbf{B}}^{+}\left[\left(\mathbf{I}-\overline{\mathbf{B}} \overline{\mathbf{B}}^{+}\right)\left\{\dot{\mathbf{x}}_{\mathbf{d}}(t)-\mathbf{A}_{\mathbf{m}} \mathbf{e}(t)-\mathbf{H}(t)\right\}\right. \\
& \left.-\overline{\mathbf{B}}^{+} \overline{\mathbf{B}}^{+} \Delta \mathbf{H}(t)-\overline{\mathbf{B}} \mathbf{K s g n}(\mathbf{s})\right] \\
& =\mathbf{s}^{\mathrm{T}}\left[-\overline{\mathbf{B}}^{+} \Delta \mathbf{H}(t)-\mathbf{K s g n}(\mathbf{s})\right]
\end{aligned}
$$

Therefore, the following condition is needed so that the time derivative of the Lyapunov function should be negative definite:

$$
(K)_{i i}>\left|\left(\bar{B}^{+} \Delta H\right)_{i}\right| \text { for } i=1, \ldots, r
$$

In other words, the magnitude of the switching gain( $\mathbf{K}$ ) must be larger than that of the term due to the TDE error.

\section{WINDUP PHENOMENON}

In this section, we will explain the windup phenomenon of TDCSA using an integral sliding surface in the presence of the saturation. Firstly, the integral element of the controller will be examined and this phenomenon occurred at linear second order system will be considered. Secondly, the action of TDC and the switching action will be examined in the presence of the saturation. Finally, the compensator for anti-windup scheme will be proposed.

\subsection{The Integral elements of TDCSA}

Two kind of an integral element exist in TDCSA using an integral sliding surface. One is the integral effect occurred by the time delay estimation of TDC. Other is the integral term of an integral sliding surface.

a) The integral effect occurred by time delay estimation [1][2]

As shown in Eq. (11), TDCSA needs the time delay values of control inputs and the derivative of states for compensating unknown dynamics and unexpected disturbance. Fig. 1 shows the block diagram of TDCSA with a saturation element in actuator. A close observation reveals important points worth mentioning: When the input is accumulated through the integral action, the control $\operatorname{input}(\mathbf{u})$ is taken for $\mathbf{u}^{*}$ owing to the saturation effect, resulting in erroneous accumulation. This erroneous accumulation of input is caused by the integration of $\mathbf{u}$ instead of $\mathbf{u}^{*}$. This phenomenon also appears in TDC[1][2]. 
Therefore, the windup phenomenon occurred by the time delay estimation is compensated by using the anti-windup scheme for TDC[1][2]. This idea leads to a simpler system, the block diagram of which is shown in Fig. 2. Clearly, the input to the actuator is made limited to $\mathbf{u}^{*}$ in advance, so that it may stay within the actuator saturation limit. In the meantime, the time-delayed value of $\mathbf{u}^{*}$ is used to produce $\mathbf{u}$, thereby preventing the erroneous accumulation.

\section{b) The integral term of the sliding variable}

Several researchers reported that, in sliding mode control using an integral sliding surface, the windup phenomenon is occurred by the integral term of the sliding variable [3][8]. More specifically, if the control input saturates, the sliding variable continues to integrate the tracking error and increases largely, thereby resulting in undesirable performance in the form of overshoot and oscillation.

We examined the two integral effects in TDCSA using an integral sliding surface. Fist of all, the anti-windup scheme for TDC compensates the windup phenomenon occurred by the time delay estimation. However, some new method is needed for compensating the windup phenomenon occurred by the integral term of the sliding variable. Now we will analyze the windup phenomenon and then propose the antiwindup scheme.

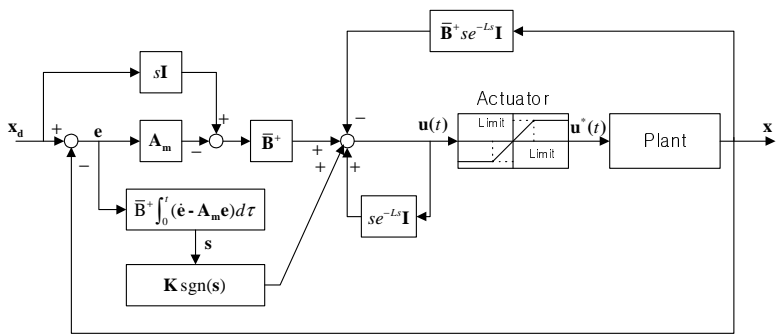

Fig.1-TDCSA TDCSA block diagram with saturation element in actuator

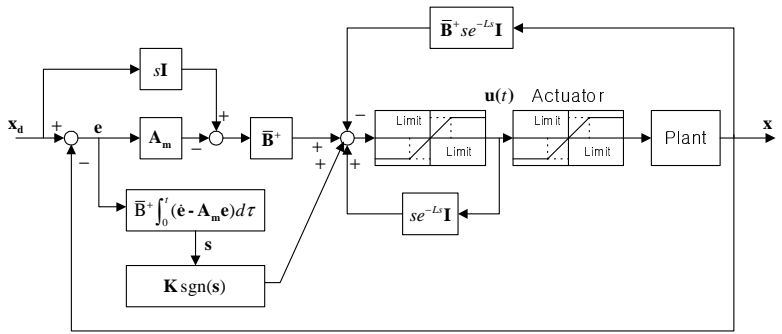

Fig.2-TDCSA block diagram with TDC anti-windup scheme

\subsection{Analysis of the windup phenomenon}

If the abovementioned anti-windup scheme of TDC is applied for compensating the windup problem, the oscillation of the control input occurred by the integral effect of time delay estimation disappears, but the sliding variable increases initially because the integral term of the sliding variable. Therefore, the windup phenomenon occurred by the time delay estimation is compensated by using the anti-windup scheme of TDC, but the windup phenomenon occurred by the integral term of the sliding variable remains.

After the control input leaves the saturation limit, we will examine how TDC and the switching action work.

\section{a) Time Delay Control}

The control objective of TDC is to make the plant follow accurately a desired error dynamics(Eq. (4)). The general solution of Eq. (4) is obtained as follows:

$\mathbf{e}(t)=e^{\mathbf{A}_{\mathbf{m}}(t-\tau)} \mathbf{e}(\tau)$, for all $\tau, t$ where $e^{\mathbf{A}_{\mathbf{m}}(t-\tau)}$ denotes a state-transition matrix. In ideal situation having no saturation element, the tracking error of TDC is to be as Eq. (15).

$\mathbf{e}(t)=e^{\mathbf{A}_{\mathrm{m}} t} \mathbf{e}(0)$

Namely initial tracking error( $\mathbf{e}(\mathbf{0}))$ goes to zero with following the desired error trajectory.

When the system has any saturation element in actuator, however, the error dynamics does not be to be as Eq. (15). In that case, after the saturation is finished $\left(t>t_{e}\right)$, TDC makes the plant follow as like Eq. (16).

$\mathbf{e}(t)=e^{\mathbf{A}_{\mathbf{m}}\left(t-t_{e}\right)} \mathbf{e}\left(t_{e}\right)$

where $t_{e}$ is the time that the saturation limit is finished; $\mathbf{e}^{*}\left(t_{e}\right)$ is an error vector at $t_{e}$. From the Eq. (16), TDC makes $\mathbf{e}^{*}\left(t_{e}\right)$ follow the desired error dynamics and moves it to zero after the saturation is finished. As shown in Fig. 3, the tracking error does not follow the desired error dynamics ((a) trajectory), but (b) trajectory. If there is not any saturation element, the tracking error becomes $\mathbf{e}\left(t_{e}\right)$ at $t=t_{e}$. When there is a saturation element, however, the tracking error becomes $\mathbf{e}^{*}\left(t_{e}\right)$ smaller than $\mathbf{e}\left(t_{e}\right)$ at $t=t_{e}$. After the saturation is finished $\left(t>t_{e}\right)$, TDC makes the tracking error follow (c) trajectory of Fig. 3 as like Eq. (16).

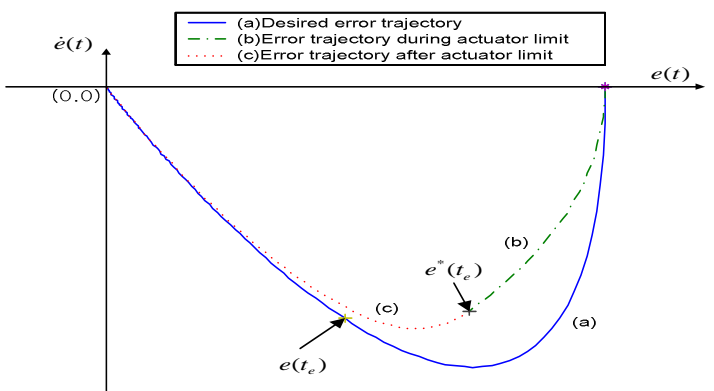

Fig.3-Phase portrait

b) Switching action

However, regardless of the saturation element, the switching action always keeps the tracking error on the sliding surface which is selected on the basis of the initial error $(e(t=0))$. More specifically, the switching action makes the tracking error become as Eq. (15)((a) trajectory of Fig. 3). As stated above, however, TDC makes the tracking error follow (c) trajectory of Fig. 3 as like Eq. (16). This means that the switching action works differently from the TDC in presence of the saturation.

In conclusion, the windup phenomenon results from the time delay estimation and the integral term of the sliding variable. In spite of the anti-windup scheme of TDC, TDC and the switching action operate differently each other so that the windup problem can not be compensated perfectly.

\subsection{Anti-Windup scheme for TDCSA}

\section{a) Anti-windup scheme}

In the presence of saturation element, we studied the windup problem resulting from the two integral effects of TDCSA using an integral sliding surface. Now, we will propose the two anti-windup methods for TDCSA; one is the abovementioned anti-windup scheme of TDC and the other is the reset sliding surface method.

The reset sliding surface method is newly proposed for compensating the effect of the integral term of sliding surface. After the saturation is finished, this method equals the sliding surface of the switching with the desired error 
dynamics of TDC. During the saturation, this method newly reconstructs the sliding surface for the switching action. More specifically, the reconstructed sliding surface is obtained from the relationship between the desired error dynamics and the sliding surface(Eq. (17)).

$\dot{\mathbf{s}}(t)=\overline{\mathbf{B}}^{+}\left[\dot{\mathbf{e}}(t)-\mathbf{A}_{\mathbf{m}} \mathbf{e}(t)\right]$

By integrating the both side terms of Eq. (17) with respect to time $(\mathrm{t})$, the sliding surface is obtained as follows:

$\mathbf{s}^{*}(t)=\overline{\mathbf{B}}^{+}\left[\mathbf{e}(t)-\int_{0}^{t} \mathbf{A}_{\mathbf{m}} \mathbf{e}(t) d \tau-\mathbf{C}\right]$

where $\mathbf{C} \in R^{n}$ is a constant vector which is selected freely. In this section, we calculate it by setting the value of $\mathbf{s}^{*}(t-L)$ to zero as follows:

$$
\begin{aligned}
\mathbf{S}^{*}(t-L) & =\overline{\mathbf{B}}^{+}\left[\mathbf{e}(t-L)-\int_{0}^{t-L} \mathbf{A}_{\mathbf{m}} \mathbf{e}(t-L) d \tau-\mathbf{C}\right]=0 \\
\mathbf{C} & =\mathbf{e}(t-L)-\int_{0}^{t-L} \mathbf{A}_{\mathbf{m}} \mathbf{e}(\tau) d \tau
\end{aligned}
$$

Fig. 4 shows the flowchart of the reset sliding surface method. The process of calculating the value of $\mathbf{C}$ only works on the saturation. After the saturation is finished, the value of $\mathbf{C}$ is to be a constant.

By adding the above two anti-windup method to TDCSA, the control law of TDCSA is as follows:

$$
\mathbf{u}(t)=\mathbf{u}^{*}(t-L)+\overline{\mathbf{B}}^{+}\left[\dot{\mathbf{x}}_{\mathbf{d}}(t)-\dot{\mathbf{x}}(t-L)-\mathbf{A}_{\mathrm{m}} \mathbf{e}(t)\right]
$$

$$
+\operatorname{Ksgn}\left(\mathbf{s}^{*}(t)\right)
$$

where $\mathbf{u}^{*}$ is the control input applied to the plant through the saturation element.

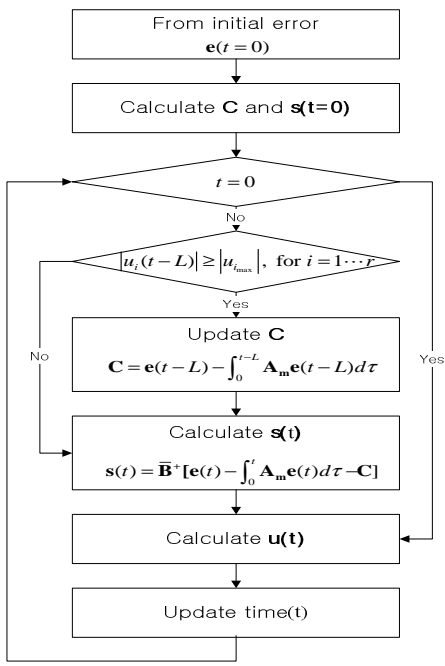

Fig.4-Flowchart of Reset sliding surface method

b) Stability Analysis of the overall system

We will analyze the stability of the overall system that has saturation elements in actuators and also is applied with TDCSA and the abovementioned two anti-windup methods.

For analyzing the stability, we divide the saturation function( $\left.\operatorname{sat}\left(u_{i}(t), u_{i_{\max }}\right)\right)$ into a linear function $\left(u_{i}(t)\right)$ and a dead-zone function $\left(\operatorname{dzn}\left(u_{i}(t), u_{i_{\max }}\right)\right)$. So the control input applied to the system through the actuator is expressed as follows:

$$
\begin{aligned}
& \mathbf{u}^{*}(t)=\operatorname{sat}(\mathbf{u}(t))=\mathbf{u}(t)-\mathbf{d z n}\left(\mathbf{u}(t), \mathbf{u}_{\max }\right) \\
& \operatorname{where~} \operatorname{sat}(\mathbf{u}(t))=\left[\begin{array}{lll}
\operatorname{sat}\left(u_{1}(t)\right) & \cdots & \operatorname{sat}\left(u_{r}(t)\right)
\end{array}\right]^{T}, \\
& \operatorname{dzn}\left(\mathbf{u}(t), \mathbf{u}_{\max }\right)=\left[\begin{array}{lll}
d z n\left(u_{1}(t), u_{1_{\max }}\right) & \cdots & d z n\left(u_{r}(t), u_{r_{\max }}\right)
\end{array}\right]^{T}
\end{aligned}
$$

where $d z n(\cdot)$ is dead-zone function; $\mathbf{u}(t)$ the control input vector; $\mathbf{u}_{\max }$ the maximum bound of the control input vector.

Combining Eq. (21) with Eq. (20), the closed error dynamics of TDCSA is as follows:

$$
\begin{aligned}
\dot{\mathbf{e}}(t) & =\mathbf{A}_{\mathbf{m}} \mathbf{e}(t)-\overline{\mathbf{B}} \overline{\mathbf{B}}^{+} \Delta \mathbf{H}(t)-\overline{\mathbf{B}} \mathbf{K} \operatorname{sgn}\left(\mathbf{s}^{*}\right) \\
& +\overline{\mathbf{B}} \mathbf{d z n}\left(\mathbf{u}(t), \mathbf{u}_{\max }\right)
\end{aligned}
$$

Note that $\overline{\mathbf{B}} \mathbf{d z n}\left(\mathbf{u}(t), \mathbf{u}_{\max }\right)$ results from the saturation element of actuator.

From the relationship (Eq. (17)) between the sliding surface and the desired error dynamics, Eq. (22) is rearranged as follows:

$$
\dot{\mathbf{s}}(t)=-\overline{\mathbf{B}}^{+} \Delta \mathbf{H}(t)-\mathbf{K s g n}\left(\mathbf{s}^{*}\right)+\mathbf{d z n}\left(\mathbf{u}(t), \mathbf{u}_{\max }\right)
$$

Lemma 1. $\|\boldsymbol{d z n}(\cdot)\|$ is satisfied with the following condition:

$$
\begin{aligned}
\left\|\operatorname{dzn}\left(\mathbf{u}(t), \mathbf{u}_{\max }\right)\right\| & \leq \beta \mathbf{u}_{\text {max }}+\beta\|\dot{\mathbf{s}}(t)\| \\
& +\beta\left\|\overline{\mathbf{B}}^{+}\right\|\|(\dot{\mathbf{x}}(t)-\dot{\mathbf{x}}(t-L))\|+\beta \mathbf{K}
\end{aligned}
$$

where $\|\cdot\|$ means the Euclidean norm.

Proof:

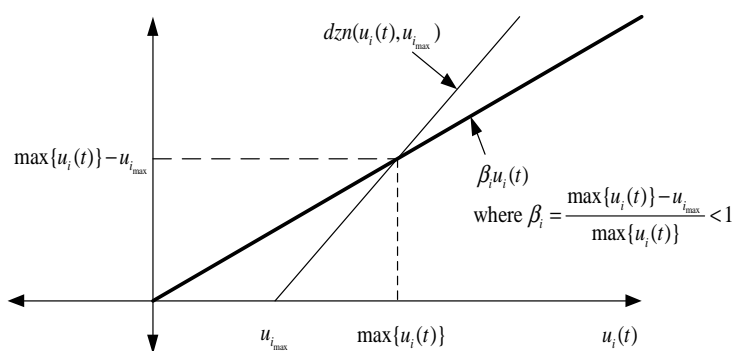

Fig.5-Linear bound of dzn() function

If $u_{i}(t)$ exists within $\left[-\max \left\{u_{i}(t)\right\}, \max \left\{u_{i}(t)\right\}\right]$ and $\beta_{i}$ becomes $1-u_{i_{\max }} / \max \left\{u_{i}(t)\right\},\left|d z n\left(u_{i}(t), u_{i_{\max }}\right)\right| \leq \beta_{i}\left|u_{i}(t)\right|$ is satisfied by Fig. 5. From the Fig. 5, $\operatorname{dzn}\left(\mathbf{u}(t), \mathbf{u}_{\max }\right)$ is bounded as the following inequality:

$$
\begin{aligned}
& \left\|\operatorname{dzn}\left(\mathbf{u}(t), \mathbf{u}_{\max }\right)\right\| \leq \beta\|\mathbf{u}(t)\| \\
& \text { where } \beta=\max _{i}\left\{1-\frac{u_{i_{\max }}}{u_{i}(t)}\right\}<1
\end{aligned}
$$

where $\beta$ is a constant which always has the value small than 1 .

By adding $\overline{\mathbf{B}}^{+} \dot{\mathbf{x}}(t)-\overline{\mathbf{B}}^{+} \dot{\mathbf{x}}(t)$ to the right side of Eq. (20), a TDCSA law is rearranged as follows:

$$
\begin{aligned}
\mathbf{u}(t)= & \mathbf{u}^{*}(t-L)+\overline{\mathbf{B}}^{+}\left[\dot{\mathbf{x}}_{\mathbf{d}}(t)-\dot{\mathbf{x}}(t-L)-\mathbf{A}_{\mathrm{m}} \mathbf{e}(t)\right] \\
& +\mathbf{K s g n}\left(\mathbf{s}^{*}(t)\right)+\overline{\mathbf{B}}^{+} \dot{\mathbf{x}}(t)-\overline{\mathbf{B}}^{+} \dot{\mathbf{x}}(t) \\
& =\mathbf{u}^{*}(t-L)+\overline{\mathbf{B}}^{+}\left[\dot{\mathbf{x}}_{\mathbf{d}}(t)-\dot{\mathbf{x}}(t)-\mathbf{A}_{\mathbf{m}} \mathbf{e}(t)\right] \\
& +\overline{\mathbf{B}}^{+}(\dot{\mathbf{x}}(t)-\dot{\mathbf{x}}(t-L))+\mathbf{K s g n}\left(\mathbf{s}^{*}(t)\right) \\
& =\mathbf{u}^{*}(t-L)+\dot{\mathbf{s}}(t)+\overline{\mathbf{B}}^{+}(\dot{\mathbf{x}}(t)-\dot{\mathbf{x}}(t-L)) \\
& +\mathbf{K s g n}\left(\mathbf{s}^{*}(t)\right)
\end{aligned}
$$

Here, the maximum value of $\mathbf{u}^{*}(t-L)$ is $\mathbf{u}_{\max }$ because the input to the actuator is made limited to $\mathbf{u}^{*}(t-L)$ in advance(Fig. 2), so the magnitude of the control input is bounded as follows: 


$$
\|\mathbf{u}(t)\| \leq \mathbf{u}_{\max }+\|\dot{\mathbf{s}}(t)\|+\left\|\overline{\mathbf{B}}^{+}\right\|\|(\dot{\mathbf{x}}(t)-\dot{\mathbf{x}}(t-L))\|+\mathbf{K}
$$

From Eq. (25) and Eq. (27), $\|\operatorname{dzn}(\cdot)\|$ is satisfied with the following condition:

$$
\begin{aligned}
& \left\|\mathbf{d z n}\left(\mathbf{u}(t), \mathbf{u}_{\text {max }}\right)\right\| \leq \beta\|\mathbf{u}(t)\| \\
& \leq \beta \mathbf{u}_{\max }+\beta\|\dot{\mathbf{s}}(t)\|+\beta\left\|\overline{\mathbf{B}}^{+}\right\|\|(\dot{\mathbf{x}}(t)-\dot{\mathbf{x}}(t-L))\|+\beta \mathbf{K}
\end{aligned}
$$

Lemma 2. If $K_{i i}=\left|\bar{B}^{+} \Delta H_{i}\right|+\eta$ and $\|\dot{\mathbf{x}}(t)-\dot{\mathbf{x}}(t-L)\| \leq \mathbf{k} L$, $\|\dot{\mathbf{s}}(t)\|$ is satisfied with the following inequality:

$$
\|\dot{\mathbf{s}}(t)\| \leq \frac{1}{1-\beta}\left[\eta+\beta \mathbf{u}_{\max }+\beta\left\|\overline{\mathbf{B}}^{+}\right\| \mathbf{k} L+\beta \mathbf{K}\right] \leq \mathbf{r}
$$

\section{Proof:}

From Eq. (23) and Lemma 1, the following inequality is obtained:

$$
\begin{aligned}
& \begin{aligned}
\|\dot{\mathbf{s}}(t)\| & \leq\left\|-\overline{\mathbf{B}}^{+} \Delta \mathbf{H}(t)-\mathbf{K} \operatorname{sgn}(\mathbf{s})\right\|+\left\|\mathbf{d z n}\left(\mathbf{u}(t), \mathbf{u}_{\max }\right)\right\| \\
& \leq\left\|-\overline{\mathbf{B}}^{+} \Delta \mathbf{H}(t)-\mathbf{K s g n}(\mathbf{s})\right\|+\beta \mathbf{u}_{\max }+\beta\|\dot{\mathbf{s}}(t)\| \\
& +\beta\left\|\overline{\mathbf{B}}^{+}\right\|\|(\dot{\mathbf{x}}(t)-\dot{\mathbf{x}}(t-L))\|+\beta \mathbf{K}
\end{aligned} \\
& \text { If } \quad K_{i i}=\left|\bar{B}^{+} \Delta H_{i}\right|+\eta \quad, \quad\|\dot{\mathbf{x}}(t)-\dot{\mathbf{x}}(t-L)\| \leq \mathbf{k} L \text { and } \beta \text { is }
\end{aligned}
$$
smaller than 1 , the following inequality is obtained:

$$
\|\dot{\mathbf{s}}(t)\| \leq \frac{1}{1-\beta}\left[\eta+\beta \mathbf{u}_{\max }+\beta\left\|\overline{\mathbf{B}}^{+}\right\| \mathbf{k} L+\beta \mathbf{K}\right] \leq \mathbf{r}
$$

\section{Theorem. The stability of the overall system}

When TDCSA using an integral sliding surface applies the system having saturation elements in actuators with the proposed two anti-windup schemes, if the following assumptions are satisfied:,

1. $\overline{\mathbf{B}}$ is satisfied with $\left\|\mathbf{I}-\mathbf{B} \overline{\mathbf{B}}^{+}\right\|<\mathbf{1}$,

2. The switching gain is selected to be $K_{i i}=\left|\bar{B}^{+} \Delta H_{i}\right|+\eta$ and

3. $\|\dot{\mathbf{x}}(t)-\dot{\mathbf{x}}(t-L)\| \leq \mathbf{k} L$,

then the overall control system is Input-to-Output Stable.

\section{Proof :}

By multiplying $\overline{\mathbf{B}}$ to the both sides of Eq. (17), Eq. (17) is arranged into the following equation:

$$
\dot{\mathbf{e}}(t)=\mathbf{A}_{\mathbf{m}} \mathbf{e}(t)+\overline{\mathbf{B}} \dot{\mathbf{s}}(t)
$$

The general solution of Eq. (32) is obtained as follows:

$$
\mathbf{e}(t)=\mathbf{e}^{\mathrm{A}_{\mathrm{m}} \mathrm{t}} \mathbf{e}_{\mathbf{m}}(\mathbf{0})+\int_{0}^{\mathrm{t}} \mathbf{e}^{\mathrm{A}_{\mathrm{m}}(\mathrm{t}-\tau)} \overline{\mathbf{B}} \dot{\mathbf{s}}(\tau) \mathbf{d} \tau
$$

where $\mathbf{A}_{\mathbf{m}}$ is a constant matrix of the desired error dynamics and $\left\|\mathbf{e}^{\mathbf{A}_{\mathrm{m}} t}\right\|$ satisfies the inequality.

$$
\left\|\mathbf{e}^{\mathbf{A}_{\mathrm{m}} t}\right\| \leq \mathbf{k}_{\mathbf{1}} \mathbf{e}^{-\alpha t}
$$

where $\alpha$ is the real part of the eigenvalue that lies nearest to the imaginary axis $\left(\min \left\{\operatorname{Re}\left[-\lambda_{i}\left(\mathbf{A}_{\mathrm{m}}\right)\right]\right\}\right)$ and $k_{1}>0$.

Because $\|\dot{\mathbf{s}}(t)\|$ is bounded by lemma 2, we obtain the following equation from Eq. (33).

$$
\begin{aligned}
& \|\mathbf{e}(t)\| \leq\left\|\mathbf{e}^{\mathbf{A}_{\mathrm{m}} \mathrm{t}}\right\|\|\mathbf{e}(\mathbf{0})\|+\|\overline{\mathbf{B}}\|\|\dot{\mathbf{s}}(\tau)\| \int_{0}^{t} \mathbf{k}_{1} \mathbf{e}^{-\alpha(t-\tau)} \mathbf{d} \tau \\
& \leq \mathbf{k}_{1} \mathbf{e}^{-\alpha \mathrm{t} t}\|\mathbf{e}(\mathbf{0})\|+\|\bar{B}\| \sup _{\tau \in[0, t]}\|\dot{s}(\tau)\| \int_{0}^{t} \mathbf{k}_{1} \mathrm{e}^{-\alpha(\mathrm{t}-\tau)} \mathbf{d} \tau \\
& \leq \mathbf{k}_{1} \mathbf{e}^{-\alpha t}\|\mathbf{e}(\mathbf{0})\|+\|\overline{\mathbf{B}}\| \sup _{\tau \in[0, t]}\|\dot{\mathbf{s}}(\tau)\| \frac{\mathbf{k}_{\mathbf{1}}}{\alpha}\left(\mathbf{1}-\mathbf{e}^{-\alpha t}\right) \\
& \leq \mathbf{k}_{1} \mathbf{e}^{-\alpha t}\|\mathbf{e}(\mathbf{0})\|+\|\overline{\mathbf{B}}\| \mathbf{r} \frac{\mathbf{k}_{1}}{\alpha}\left(\mathbf{1}-\mathrm{e}^{-a \mathrm{t}}\right)
\end{aligned}
$$

From Eq. (35), regardless of time, the tracking error is bounded so that the overall system is Input-to-output stable [9][10].

\section{EXPERIMENTS}

To verify the effectiveness of the proposed compensators, experiments have been made on 2 degree of freedom SCARA-type robot, where the link lengths are $1_{1}=350 \mathrm{~mm}$ and $\mathrm{l}_{2}=200 \mathrm{~mm}$ and the link weights are $\mathrm{m}_{1}=11.17 \mathrm{~kg}$ and $\mathrm{m}_{2}=6.87 \mathrm{~kg}$. At each joint, a Harmonic Drive (with gear reduction ratio of $100: 1$ for joint 1 and $80: 1$ for joint 2) is used. For implementing the proposed control algorithm, we used the MC68040 controller installed with real-time OS, VxWorks. Here, the sampling frequency is selected as 250 $\mathrm{Hz}$.

\subsection{Controller Design}

The dynamics of the planar robot is as follows:

$\mathbf{M}(\boldsymbol{\theta}) \ddot{\boldsymbol{\theta}}(t)+\mathbf{V}(\boldsymbol{\theta}, \dot{\boldsymbol{\theta}})+\mathbf{D}(\boldsymbol{\theta}, \dot{\boldsymbol{\theta}})=\boldsymbol{\tau}(t)$

where $\boldsymbol{\theta}=\left[\begin{array}{ll}\theta_{1} & \theta_{2}\end{array}\right]^{T}$ denotes the joint vector; $\boldsymbol{\tau}(t)=\left[\begin{array}{ll}\tau_{1} & \tau_{2}\end{array}\right]^{T}$ joint torque vector.

From Eq.(36), a TDCSA law is obtained for the planar robot as follows:

$$
\begin{aligned}
\tau_{i}(t) & =\tau_{i}(t-L)+\bar{M}_{i}\left\{\ddot{\theta}_{d i}-\ddot{\theta}_{i}(t-L)+2 \varsigma_{i} w_{n i} \dot{e}_{i}\right. \\
& \left.+w_{n i}^{2} e_{i}\right\}+K_{i} \operatorname{sgn}\left(s_{i}\right), \text { for } i=1,2
\end{aligned}
$$

The value of the gains was selected to be $w_{n 1}=w_{n 2}=8[\mathrm{rad} / \mathrm{s}], \quad \varsigma_{1}=\varsigma_{2}=1, \quad K_{1}=0.05, \quad K_{2}=0.01$, $\bar{M}_{1}=0.005, \bar{M}_{2}=0.001$.

\subsection{Experimental Setup}

Fig. 6 shows the experimental setup for each axis. The saturation limit is set $\left|\tau_{1}(t)\right| \leq 3.0 \mathrm{~V}=0.5751 \mathrm{Nm}$ for joint 1 and $\left|\tau_{2}(t)\right| \leq 1.5 \mathrm{~V}=0.1356 \mathrm{Nm}$ for joint 2 .

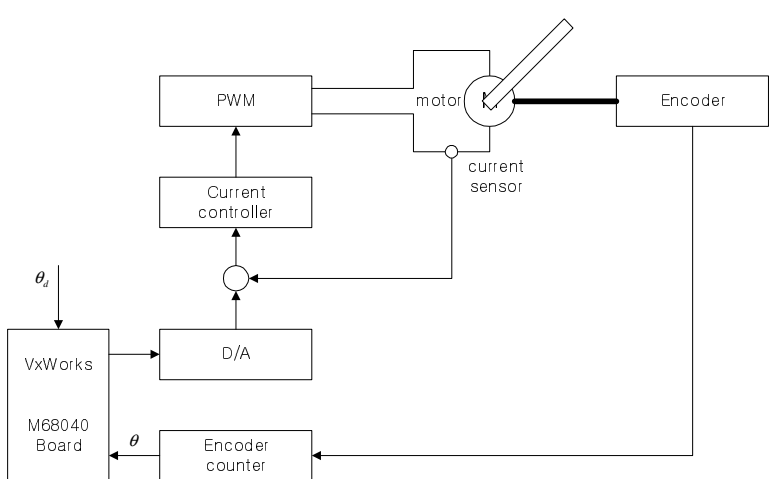

Fig.6-Experimental setup for each axis 


\subsection{Experimental Results}

For observing the windup phenomenon of TDCSA using an integral sliding surface, first of all, we experiment on the step input trajectory : $\theta_{d 1}=45$ [degree], $\theta_{d 2}=90$ [degree] .

Fig. 7 shows the experimental results in situation of applying the only anti-windup scheme of TDC for compensating the windup phenomenon. As shown in Fig. 7, the windup effect is increased as the desired response speed, which is expressed in terms of $w_{n}$, is increased. To be more specific, as the desired response $\operatorname{speed}\left(w_{n}\right)$ is increased, the integral term of sliding variables is increased so that the windup effect is increased, thereby causing overshoot in the response.

Fig. 8 shows the experimental result with TDC anti-windup scheme and the reset sliding surface method for compensating the windup effect. As shown in Fig. 8, regardless of the $w_{n}$, the sliding variable does not increased excessively so that any overshoot does not appear in the response. This means that the reset sliding surface compensates well the windup effect occurred by the integral term of the sliding surface well.

Through the above experiments, we examined that the windup phenomenon appears in TDCSA using an integral sliding surface and the proposed anti-windup schemes compensate it well.
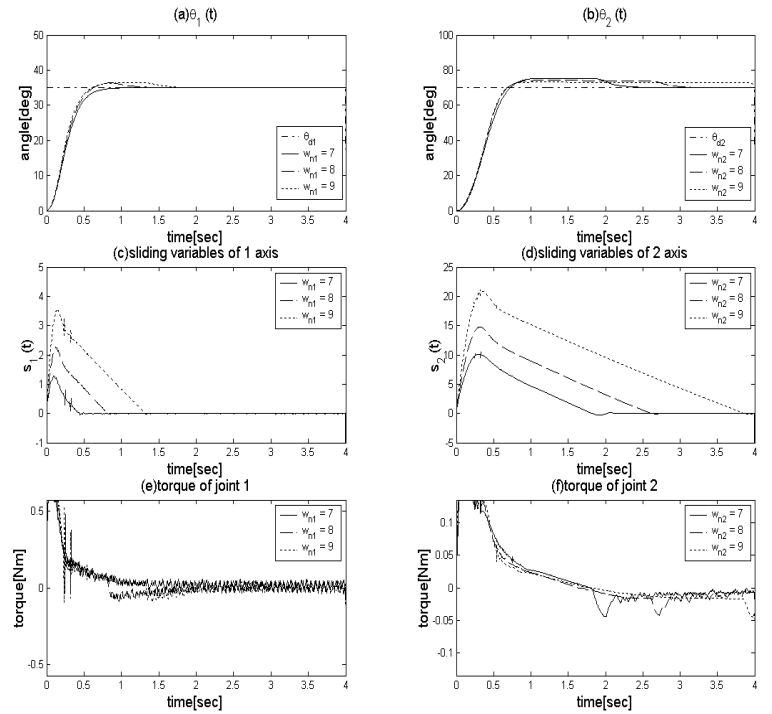

Fig.7-Experimental result with only anti-windup scheme of $T D C$
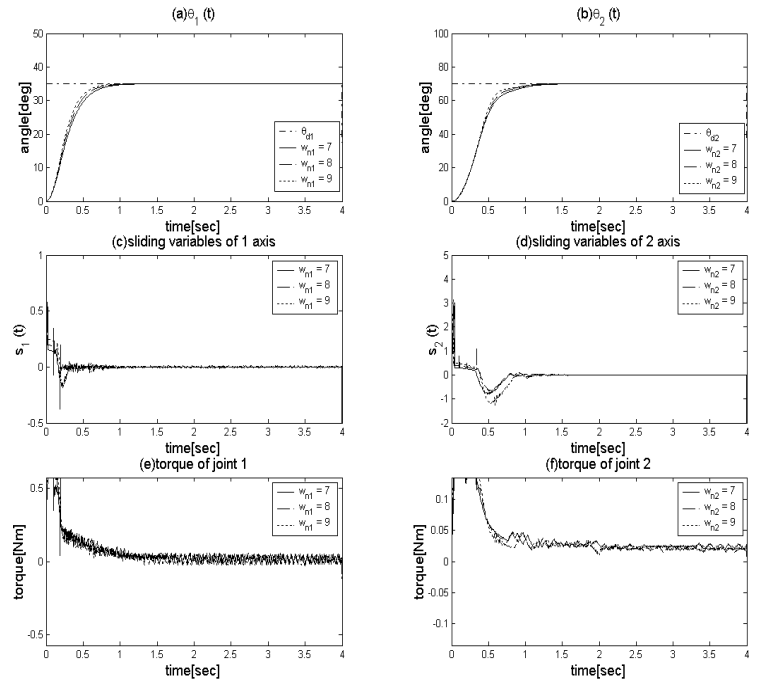

Fig.8-Experimental result with anti-windup scheme of TDC and reset sliding surface method

\section{CONCLUSION}

In this paper, we have reported the windup phenomenon associated with TDCSA using an integral sliding surface, presented its causes, and proposed its remedies. Specifically, we have shown that the windup phenomenon results from the inherent two integral effects in TDCSA using an integral sliding surface: the integral effect of the time delay estimation and the integral term of the integral sliding surface.

So we have proposed the anti-windup schemes for two integral effects, respectively. For the integral effect of the time delay estimation, we proposed the existing antiwindup scheme of TDC. However, this scheme can not compensates the windup effect occurred by the integral term of the sliding surface, and then we proposed the reset sliding surface for the windup effect of the sliding variable.

Through the experiments on a SCARA-type robot, we showed that, in TDCSA using an integral sliding surface, there exists the windup phenomenon and the proposed compensators are effective enough to handle the windup phenomenon well.

Finally, the proposed anti-windup scheme will be useful to SMC using an integral sliding surface.

\section{REFERENCES}

(1). Chang, P. H. and Park, S. H., "The development of anti-windup scheme and stick-slip compensator for time delay control", Proc. of the American Control Conference, pp. 3629-3633, 1998

(2). Chang, P. H., Park, S. H. and Lee, S. U.,"Development of Anti-Windup Method for Time Delay Control," Trans. of the Korea Society of Mechanical Eng., vol. 18, Iss. 10, pp. 2616-2628, 1994.

(3). Cho, Dan, Yoshifumi Kato and Darin Spilman, "Sliding Mode and Classical Controllers in Magnetic Levitation System”, IEEE Control Systems, pp. 42-48, 1993

(4). Hsia, T. C. and Gao, L. S., "Robot Manipulator control using decentralized linear time-invariant time-delayed joint controllers", Proc. of IEEE Conference on Robotics and Automation, pp. 2070-2075, 1990

(5). Lee, J.H. and Youn, M.J. "A New Integral Variable Structure Regulation Controller for Robot Manipulators with Accurately Predetermined Output", ISIE'99 Proc. of the IEEE International Symposium on Industrial Electronics, pp. 336-341, 1999

(6). Lee, S. U. and Chang, P. H., "Control of a heavy-duty Robotic Excavator using Time Delay Control with Integral Sliding Surface", Control Engineering Practice, vol. 10, no. 7, pp. 697-711, 2002

(7). Park, S. H., "Robust Controller and Observer using Synthesis of TDE and Switching Action", Ph. D. thesis, Korea Institute of Advanced Science and Technology, 2000.

(8). Slotine, J.-J. E. and Li, J.-J. E.,Applied Nonlinear Control, Prentice-Hall International Editions, 1991

(9). Sontag, E. D. "Smooth stabilization implies coprime factorization," IEEE Trans. on Automatic Control, Vol. 34, pp. 435-443, 1989

(10). Sontag, E. D. and Wang, Y., "New Characterizations of Input-to-State Stability", IEEE Trans. on Automatic Control, vol. 41, n. 9, pp. 1283-1294, 1996

(11). Youcef-Toumi, K. and Ito, O., "A time delay controller for systems with unknown dynamics", ASME Journal of Dynamic System, Measurement and Control, Vol. 112, pp. 133-141, 1990 